\title{
Investigation of Possible Herb-Drug Interactions for the Treatment of Cardiovascular Diseases
}

\author{
${ }^{1}$ State Key Laboratory of Quality Research in Chinese Medicine, \\ Institute of Chinese Medical Sciences, University of Macau, Macau \\ Special Administrative Region, China \\ 2 School of Chemistry and Chemical Engineering, Chongqing \\ University, Chongqing, China \\ Semin Thromb Hemost 2019;45:548-551.
}

Huali L. Zuo, MSc ${ }^{1}$ Fengqing Q. Yang, $\mathrm{PhD}^{2}$ Yuanjia J. Hu, $\mathrm{PhD}^{1}$

\begin{abstract}
Address for correspondence Yuanjia J. Hu, PhD, State Key Laboratory of Quality Research in Chinese Medicine, Institute of Chinese Medical Sciences, University of Macau, Macau Special Administrative Region 999078, China (e-mail: yuanjiahu@um.edu.mo).
\end{abstract}

Fengqing Q. Yang, PhD, School of Chemistry and Chemical Engineering, Chongqing University, Chongqing 401331, China (e-mail: fengqingyang@cqu.edu.cn).

used for invigorating blood circulation and eliminating stasis. ${ }^{11,12} \mathrm{~A}$ pairing (a basic unit of complex herbal formulae) of the two herbs was one of the most frequently compatible herbal pairs in best-selling herbal formulae released by the China Association of Chinese Medicine in 2017. ${ }^{13}$ Therefore, our investigation employs the herbal pair (DS - CX) to analyze the mechanism of action and possible interaction with Western drugs. Moreover, to understand the intrinsic herb - drug interaction on the molecular level, this investigation uses the network of pharmacological approach ${ }^{14}$ to integrate the abundant data which have accumulated from previous research on herbal medicines and current approved anti-CVD drugs. For detailed methods, please refer to the Appendix (also refer to -Fig. 1 and -Tables 1, 2).

In this investigation, the "target - (pathway) - target" network clearly shows that DS - CX interacts with the targets of anti-CVD Western drugs. DS - CX may interact with 56 (33.9\%) of targets of anti-CVD Western drugs. Totally, DS - CX's 384 compounds may affect 567 biological molecules. Meanwhile, it is of significance to specify the kind of interactions in the specific pathways, especially the main thrombotic pathways. Our investigation found that two (prostaglandin-endoperoxide synthase 1 [PTGS1], integrin subunit alpha 2b [ITGA2B]; 22.2\%) out of nine Western medicine targets on platelet activation are involved in target sets of DS - CX. Similarly, for the pathway of vascular smooth muscle contraction, there are 6 of 17 drug targets (adenosine A2a receptor [ADORA2A], ADORA2B, adrenoceptor alpha 1A [ADRA1A], ADRA1B, ADRA1D, potassium calcium-activated channel subfamily $M$ alpha 1 [KCNMA1]; 35.3\%) are relevant to DS - CX. In addition, for the pathway of complement and coagulation cascades, the corresponding number is 4 (F2, F10, Serpin [serine protease inhibitors] family E member 1 [SERPINE1], plasminogen activator, urokinase [PLAU]; 36.4\%) out of 11 . Refer to the Appendix for published online June 13, 2019
Issue Theme Editorial Compilation VII; Guest Editors: Emmanuel J. Favaloro, PhD, FFSc (RCPA), and Giuseppe Lippi, MD.
Copyright $\odot 2019$ by Thieme Medical Publishers, Inc., 333 Seventh Avenue, New York, NY 10001, USA. Tel: +1(212) 584-4662.
DOI https://doi.org/ 10.1055/s-0039-1692211. ISSN 0094-6176. 
more detailed results of network analysis. Higher overlapping intensity between pharmaceutical and herbal targets may mean higher risk in clinical practice of Western drugs.

For example, the verified corresponding targets of aspirin include prostaglandin $\mathrm{G} / \mathrm{H}$ synthase 1 (COX-1 [Cyclooxygenase-1]; gene name: PTGS1) and prostaglandin $\mathrm{G} / \mathrm{H}$ synthase 2 (COX-2; gene name: PTGS2). ${ }^{15,16}$ COX-1 mainly contributes to the arachidonic acid metabolism pathway and the platelet activation pathway. COX-2 is mainly involved in the arachidonic acid metabolism pathway directly relevant to thrombosis and the vascular endothelial growth factor (VEGF) signaling pathway relevant to thrombosis indirectly. COX-1 and COX-2 might be targeted by senkyunolide B, senkyunolide $C$, and senkyunolide $E$ from $C X$, and phenanthraquinones from DS, including tanshinone I, 1-dehydrotanshinone IIA, neocryptotanshinone, przewaquinone $\mathrm{A}$, isotanshinone I, nortanshinone, etc. Previous studies have partially supported the potential effects of DS and CX on COX-1 and COX-2. ${ }^{11,12,17}$

With the wide prevalence of active herbal medicines, it is necessary to add an understanding of the biological mechanism of herb - drug interaction. In general, this
(A)

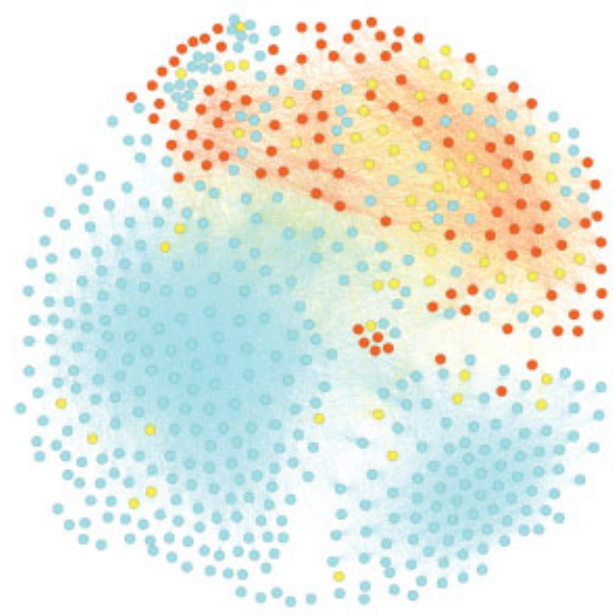

(C)

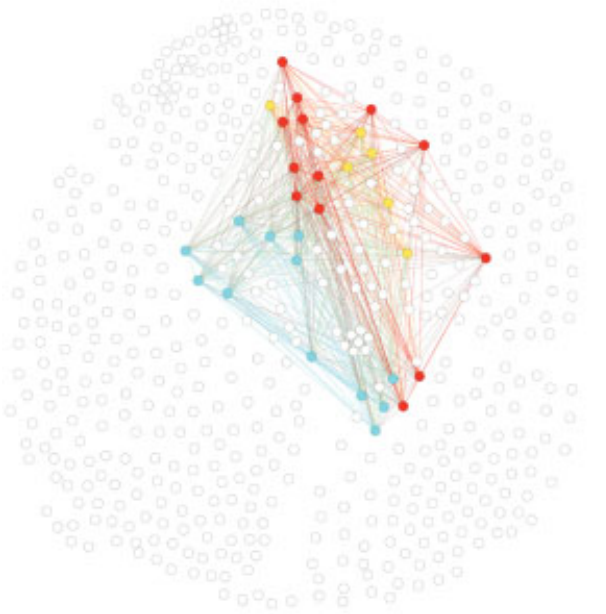

(B)

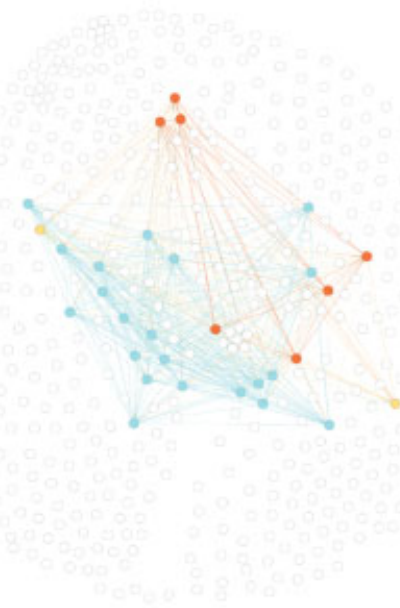

(D)

\section{Anti-CVD drug targets}

The common targets of anti-CVD drugs and DS-CX DS-CX targets Targets irrelevant to specific pathways

Fig. 1 The TPT (target - [pathway] - target) networks; (A) whole network, (B) subnetwork in the platelet activation pathway, (C) subnetwork in the vascular smooth muscle contraction pathway, (D) subnetwork in the complement and coagulation cascades pathway. CVD, cardiovascular disease; DS-CX, danshen-chuanxiong. 
Table 1 A general comparison of conventional anti-CVD drugs and DS - CX

\begin{tabular}{|l|l|l|l|l|l|}
\hline & $\begin{array}{l}\text { Western } \\
\text { drugs (W) }\end{array}$ & $\begin{array}{l}\text { Danshen } \\
(\text { DS })\end{array}$ & $\begin{array}{l}\text { Chuanxiong } \\
(\text { CX) }\end{array}$ & DS - CX & JI \\
\hline Compounds & 217 & 188 & 220 & 384 & $0.005^{\mathrm{a}}$ \\
\hline Targets & 165 & 439 & 339 & 567 & 0.339 \\
\hline
\end{tabular}

Abbreviations: CVD, cardiovascular disease; DS-CX, danshen-chuanxiong; FDA, Food and Drug Administration; Jl, Jaccard's index.

${ }^{a}$ Adenosine is the only common compound of anti-CVD drugs and DS - CX. It was approved in 1989 by FDA for cardiac therapy.

Table 2 Target-based interaction between anti-CVD drugs and DS - CX

\begin{tabular}{|l|l|l|l|}
\hline & Anti-CVD drug targets & Common targets & JI \\
\hline Whole network - Fig. 1A & 165 & 56 & 0.339 \\
\hline Subnetwork - Fig. 1B & 9 & 2 & 0.222 \\
\hline Subnetwork - Fig. 1C & 17 & 6 & 0.353 \\
\hline Subnetwork - Fig. 1D & 11 & 4 & 0.364 \\
\hline
\end{tabular}

Abbreviations: CVD, cardiovascular disease; JI, Jaccard's index.

kind of herb - drug interaction implies that patients simultaneously taking anti-CVD Western drugs and DS - CX may be exposed to additional risks caused by accumulated effects on common herb - drug targets. More importantly, this kind of risk is not usually visible and difficult for clinical physicians to observe due to wide herbal utilization as undisclosed self-medication. In this context, herb - drug relationships are worthy of further exploration in the future, especially from experimental and clinical perspectives.

\section{Authors' Contributions}

Y.J.H. and F.Q.Y. conceived and designed the study; H.L.Z. analyzed data, performed the network analysis, and drafted the manuscript; Y.J.H. and F.Q.Y. revised the manuscript. All authors have read and approved the final manuscript.

\section{Funding}

This work was financially supported by the Science and Technology Development Fund of Macao Special Administrative Region and the University of Macau for projects 013-2015-A1 and MYRG2016-00144-ICMS-QRCM.

\section{Conflicts of Interest}

The authors have no conflicts of interest to declare.

\section{References}

1 McEwen BJ. The influence of herbal medicine on platelet function and coagulation: a narrative review. Semin Thromb Hemost 2015; 41(03):300-314

2 Maione F, Mascolo N. Danshen and the cardiovascular system: New advances for an old remedy. Semin Thromb Hemost 2016;42 (03):321-322

3 Lüscher TF. Improving outcome in acute coronary syndromes: ischaemic conditioning, antithrombosis and bleeding, and inflammasome antagonism. Eur Heart J 2017;38(11):763-766

4 Liperoti R, Vetrano DL, Bernabei R, Onder G. Herbal medications in cardiovascular medicine. J Am Coll Cardiol 2017;69(09): 1188-1199
5 Tachjian A, Maria V, Jahangir A. Use of herbal products and potential interactions in patients with cardiovascular diseases. J Am Coll Cardiol 2010;55(06):515-525

6 Chen C, Yang FQ, Zhang Q, Wang FQ Hu YJ, Xia ZN. Natural products for antithrombosis. Evid Based Complement Alternat Med 2015;2015:876426

7 Li L, Zhou X, Li N, Sun M, Lv J, Xu Z. Herbal drugs against cardiovascular disease: traditional medicine and modern development. Drug Discov Today 2015;20(09):1074-1086

8 Samuels N. Herbal remedies and anticoagulant therapy. Thromb Haemost 2005;93(01):3-7

9 McEwen BJ. Comment on "Danshen and the cardiovascular system: new advances for an old remedy.". Semin Thromb Hemost 2016;42(03):323-324

10 Chen XWB, Sneed KB, Pan SY, et al. Herb-drug interactions and mechanistic and clinical considerations. Curr Drug Metab 2012; 13(05):640-651

11 Chen Z, Zhang C, Gao F, et al. A systematic review on the rhizome of Ligusticum chuanxiong Hort. (Chuanxiong). Food Chem Toxicol 2018;119:309-325

12 Chen X, Guo J, Bao J, Lu J, Wang Y. The anticancer properties of Salvia miltiorrhiza Bunge (Danshen): a systematic review. Med Res Rev 2014;34(04):768-794

13 Li G, Cheng YH, Guo YB, et al. Report on scientific and technological competitiveness of big brand traditional Chinese medicine (2017 edition). Zhongguo Xiandai Zhongyao 2018;20(01):6-13

14 Zuo H, Zhang Q Su S, Chen Q, Yang F, Hu Y. A network pharmacology-based approach to analyse potential targets of traditional herbal formulas: an example of Yu Ping Feng decoction. Sci Rep 2018;8(01):11418

15 Knox C, Law V, Jewison T, et al. DrugBank 3.0: a comprehensive resource for 'omics' research on drugs. Nucleic Acids Res 2011;39 (Database issue, suppl. 1):D1035-D1041

16 Ursu O, Holmes J, Knockel J, et al. DrugCentral: online drug compendium. Nucleic Acids Res 2017;45(D1):D932-D939

17 Fang J, Little PJ, Xu S. Atheroprotective effects and molecular targets of tanshinones derived from herbal medicine Danshen. Med Res Rev 2018;38(01):201-228

18 Guo Y, Nie Q, MacLean AL, Li Y, Lei J, Li S. Multiscale modeling of inflammation-induced tumorigenesis reveals competing oncogenic and onco-protective roles for inflammation. Cancer Res 2017;77(22):6429-6441

19 Li S. Mapping ancient remedies: applying a network approach to traditional Chinese medicine. Science 2015;350(6262):S72-S74 
20 Chen CYC. TCM Database@Taiwan: the world's largest traditional Chinese medicine database for drug screening in silico. PLoS One 2011;6(01):e15939

21 Wang JF, Zhou H, Han LY, Chen X, Chen YZ, Cao ZW. Traditional Chinese medicine information database. Clin Pharmacol Ther 2005;78(01):92-93

$22 \mathrm{Ye} \mathrm{H}, \mathrm{Ye} \mathrm{L}$, Kang H, et al. HIT: linking herbal active ingredients to targets. Nucleic Acids Res 2011;39(Database issue, suppl. 1):D1055-D1059

$23 \mathrm{Ru}$ J, Li P, Wang J, et al. TCMSP: a database of systems pharmacology for drug discovery from herbal medicines. J Cheminform 2014;6(13):13

\section{Appendix-Methods and Results}

Network pharmacology has been widely used to explain complex biological system and herbal medications. ${ }^{18,19}$ This investigation employed the approach of network pharmacology to explain the possible biological mechanism of herb drug interaction at the drug targets of action. First, the data of compounds of danshen-chuanxiong (DS - CX) were preliminarily collected from chemical databases, including TCM database@Taiwan, ${ }^{20}$ traditional Chinese medicine-information database (TCM-ID), ${ }^{21}$ Herbal Ingredients' Targets Database (HIT), ${ }^{22}$ and traditional Chinese medicine systems pharmacology database (TCMSP), ${ }^{23}$ together with the supplements from PubMed literature. The data of targets or putative targets of DS - CX compounds were obtained from PubChem, ${ }^{24}$ TCMSP, ${ }^{23}$ and similarity ensemble approach (SEA). ${ }^{25}$ Moreover, we collected information around drugs used for the treatment of cardiovascular diseases (CVDs), mainly including anti-CVD small molecules and biological products approved by the U.S. Food and Drug Administration (FDA) by September $2017 .^{26}$ The data of targets of these conventional drugs were extracted from the databases of Drugbank ${ }^{15}$ and Drugcentral. ${ }^{16}$ The enrichment analysis of targets of DS - CX and conventional drugs was carried out by STRING ${ }^{27}$ to acquire the target - pathway associations.

Based on the target - pathway associations, a "target (pathway) - target" (TPT) network was constructed with the reference to our previous work. ${ }^{14}$ In this TPT network, a node represents a DS - CX's or pharmaceutical target distinguished by different colors, and an edge indicates that both of connected nodes are involved in at least one of the same pathways. By highlighting targets relevant to specific pathways and
24 Kim S, Thiessen PA, Bolton EE, et al. PubChem substance and compound databases. Nucleic Acids Res 2016;44(D1):D1202-D1213

25 Keiser MJ, Roth BL, Armbruster BN, Ernsberger P, Irwin JJ, Shoichet BK. Relating protein pharmacology by ligand chemistry. Nat Biotechnol 2007;25(02):197-206

26 Santos R, Ursu O, Gaulton A, et al. A comprehensive map of molecular drug targets. Nat Rev Drug Discov 2017;16(01):19-34

27 Szklarczyk D, Franceschini A, Kuhn M, et al. The STRING database in 2011: functional interaction networks of proteins, globally integrated and scored. Nucleic Acids Res 2011;39(Database issue, suppl. 1):D561-D568

neglecting irrelevant ones, the TPT network can be shown as various subnetworks specific to different pathways.

-Fig. 1A shows the whole TPT network and various TPT subnetworks specific to different anti-thrombotic pathways, that is, the platelet activation pathway ( $\mathbf{F i g . ~ 1 B ) , ~ t h e ~ v a s c u l a r ~}$ smooth muscle contraction pathway (-Fig. 1C), and the complement and coagulation cascades pathway (-Fig. 1D).

As identified in -Fig. 1A, there exists a large number of biological molecules relevant to DS - CX; more importantly, many of them are also targets of conventional anti-CVD drugs. This type of overlap seems to be more distinct in the vascular smooth muscle contraction pathway and the complement and coagulation cascades pathway in comparison with the platelet activation pathway, as identified in - Fig. 1B-D. In order to measure quantitatively, we established an adjusted Jaccard's index (JI) which denotes how many percentages of compounds or targets of anti-CVD drugs are covered by DS - CX. In - Table 1, values in columns "W," "DS," "CX," and "DS-CX" represent the number of corresponding compounds or targets. As - Table 1 shows, there is only one common compound of anti-CVD drugs and DS - CX, but targets between them have a high overlap.

Moreover, - Table 2 shows target-based interaction between anti-CVD drugs and DS - CX in different thrombotic pathways. Values in the middle of the two columns indicate the corresponding number of anti-CVD drug targets or common targets, which are used to calculate the JI. The relatively higher JI values in - Fig. 1C, D further demonstrate the observation in - Fig. 1 that herb - drug interaction between anti-CVD drugs and DS - CX may be more serious in the vascular smooth muscle contraction pathway and the complement and coagulation cascades pathway. 